\title{
AngelCast: Cloud-based Peer-Assisted Live Streaming Using Optimized Multi-Tree Construction*
}

\author{
Raymond Sweha \\ Boston University \\ remos@cs.bu.edu
}

\author{
Vatche Ishakian \\ Boston University \\ visahak@cs.bu.edu
}

\author{
Azer Bestavros \\ Boston University \\ best@cs.bu.edu
}

\begin{abstract}
Increasingly, commercial content providers (CPs) offer streaming and IPTV solutions that leverage an underlying peerto-peer (P2P) stream distribution architecture. The use of $\mathrm{P} 2 \mathrm{P}$ protocols promises significant scalability and cost savings by leveraging the local resources of clients - specifically, uplink capacity. A major limitation of $\mathrm{P} 2 \mathrm{P}$ live streaming is that playout rates are constrained by the uplink capacities of clients, which are typically much lower than downlink capacities, thus limiting the quality of the delivered stream. Thus, to leverage $\mathrm{P} 2 \mathrm{P}$ architectures without sacrificing the quality of the delivered stream, CPs must commit additional resources to complement those available through clients. In this paper, we propose a cloud-based service - AngelCast that enables CPs to elastically complement P2P streaming "as needed". By subscribing to AngelCast, a CP is able to deploy extra resources ("angels"), on-demand from the cloud, to maintain a desirable stream (bit-rate) quality. Angels need not download the whole stream (they are not "leachers"), nor are they in possession of it (they are not "seeders"). Rather, angels only relay (download once and upload as many times as needed) the minimal possible fraction of the stream that is necessary to achieve the desirable stream quality, while maximally utilizing available client resources. We provide a lower bound on the minimum amount of angel capacity needed to maintain a certain bit-rate to all clients, and develop a fluid model construction that achieves this lower bound. Realizing the limitations of the fluid model construction - namely, susceptibility to potentially arbitrary start-up delays and significant degradation due to churn - we present a practical multi-tree construction that captures the spirit of the optimal construction, while avoiding its limitations. In particular, our AngelCast protocol achieves near optimal performance (compared to the fluidmodel construction) while ensuring a low startup delay by maintaining a logarithmic-length path between any client
\end{abstract}

* This research was supported in part by NSF awards \#0720604, \#0735974, \#0820138, \#0952145, and \#1012798.

Permission to make digital or hard copies of all or part of this work for personal or classroom use is granted without fee provided that copies are not made or distributed for profit or commercial advantage and that copies bear this notice and the full citation on the first page. To copy otherwise, to republish, to post on servers or to redistribute to lists, requires prior specific permission and/or a fee.

MMSys'12, February 22-24, 2012, Chapel Hill, North Carolina, USA.

Copyright 2012 ACM 978-1-4503-1131-1/12/02 ...\$10.00. and the provider, and while gracefully dealing with churn by adopting a flexible membership management approach. We present the blueprints of a prototype implementation of AngelCast, along with experimental results confirming the feasibility and performance potential of our AngelCast service when deployed on Emulab and PlanetLab.

\section{INTRODUCTION}

Streaming high-quality (HQ) video content over the Internet is becoming a standard expectation of clients, posing significantly different challenges for today's content providers (CPs) such as Netflix, Hulu, or IPTV, compared to the challenges associated with the best-effort delivery of low-quality streaming through CPs such as YouTube and Facebook. For example, Netflix reported last year that it is delivering streams at rates between $1.4 \mathrm{Mbps}$ and $2.8 \mathrm{Mbps}$ [.

Motivation and Scope: To be able to deliver streams with a high bit-rate, content providers resort to Content Delivery Networks (CDNs) to deliver their content. Those CDN's, in turn, started to tap into client upload bandwidth through the use of $\mathrm{P} 2 \mathrm{P}$ architectures to alleviate some of their own costs. Examples of peer-assisted content distribution systems include Akamai's Netsession [1, Octoshape Infinite Edge [2, Pando 3], and BitTorrent DNA 4]. The problem with pure $\mathrm{P} 2 \mathrm{P}$ architectures is that the current offerings by ISPs provide significantly higher download rate than upload rate. For example, Verizon FiOS offers three packages, 15/5 Mbps downlink/uplink capacity, 25/25 Mbps, and 50/20 Mbps. Comcast Xfinity offers $50 / 10,22 / 5,16 / 2$, or $12 / 2$ Mbps. Those examples illustrate an inherent shortcoming in pure P2P systems: The persistent gap between the average downlink and the average uplink capacity of peers creates a persistent gap between the clients expectation in terms of download rate and what the uplink capacities of their peers allows. This gap can be overlooked in typical P2P file sharing or VoD when some peers linger in the swarm after finishing downloading, thus allowing other clients to utilize them as seeders. In the case of live streaming, due to its real-time continuing nature, clients at any point in time will have significantly higher average download bandwidth than their average uplink capacity. To deal with this, proposed peer-assisted streaming systems rely on dedicated provider servers (or "seeders" in P2P jargon), which must download the live stream first before uploading it to clients.

Paper Contributions: In this paper we propose a cloudbased "live stream-acceleration" service, AngelCast. By subscribing to AngelCast, a CP is assured that its clients would be able to download the stream at the desired rate with- 
out interruptions, while maximally leveraging the benefits from P2P delivery. AngelCast achieves this by (1) enlisting special servers from the cloud, called angels ${ }^{1}$ which can supplement the gap between the average client uplink capacity and the desirable stream bit-rate. Angels are more efficient than seeders as they do not download the whole stream, but rather they download only the minimum fraction of the stream that enables them to fully utilize their upload bandwidth. In our architecture, the capacity that otherwise would have been wasted in downloading the full live stream to the servers can be channelled to help the clients directly; (2) choreographing the connectivity between nodes (clients and angels) to form optimized end-system multi-trees for peers to exchange stream content; and (3) handling clients dynamic arrival and departure.

We present theoretical results that establish the minimum amount of angel capacity needed to allow all clients to download at a desirable rate, as a function of their downlink/uplink capacities. We show that this lower bound is tight by choreographing the connectivity of nodes in such a way that the optimal bound is achieved under a fluid model.

A good live streaming system would also minimize the start-up delay needed to assure continued service. We prove that the start-up delay is zero under the fluid model, but that the optimal construction leads to a start-up delay that is linear in the number of clients when relaxing the fluid model assumption. Moreover, an optimal construction may require building a full mesh between clients (with no bound on node degrees). These reasons lead us to develop a more practical approach that utilizes almost the minimal amount of angel capacity (predicted under the fluid model), while also ensuring that the start-up delay is logarithmic in the number of clients. Our practical approach relies on dividing the stream into substreams, each of which is disseminated along a separate tree. To download the stream, each client subscribes to all the substreams, whereas angels subscribe only to one substream, allowing them to upload at full capacity while not wasting too much upload bandwidth in downloading the whole live stream. The idea of splitting the stream into substreams was proposed in prior work, most notably in SplitStream [10] and 17]. In the related work section, we discuss what we learned from those proposed techniques, and how we avoided some of their shortcomings.

Another limitation of the fluid (optimal) choreography is that it does not consider issues of churn due to node arrival and departures. We address this limitation by incorporating membership management capabilities that ensure uninterrupted service with minimum startup delay. We achieve this by ensuring that the trees used in our construction are well balanced, and by avoiding degenerate cases. Our cloudbased service provides a registrar that collects information about clients, making fast membership management decisions that ensure smooth streaming.

We discuss the architecture of our proposed AngelCast system, and evaluate a prototype implementation against SopCast [5] - a commonly use P2P streaming client. The experimental results carried out on Emulab and PlanetLab show the utility of angels and the effectiveness of our choreographed live stream distribution.

The remainder of this paper is organized as follows: In

${ }^{1}$ We introduced the notion of angels in prior work 27, where angels were used for a different objective - namely to minimize the bulk download time for a fixed group of clients.
Section 2, we present the theoretical model that bounds the minimum amount of angel upload bandwidth needed to deliver the stream to all clients with the required bit-rate. We also present an optimal fluid construction that achieves that bound and compute the start-up delay associated with it. We conclude that section by highlighting the effectiveness of using angels over using seeders for live streaming. In Section 3 we present our practical construction that avoids the impracticalities of the optimal construction by relaxing the fluid assumption and bounding the node degree. In Section 4. we present our AngelCast service architecture including the membership management techniques and the design of our protocol. In Section 5, we experimentally evaluate our AngelCast prototype against SopCast in Emulab and PlanetLab. In Section 6, we review the related work. We conclude in Section 7 with a summary of results and directions for future research.

\section{THEORETICAL BOUNDS}

We adopt the Uplink Sharing Model (USM) presented by Mundinger in [18], wherein each client is defined solely by its uplink and downlink capacities. The client is free to divide its uplink/downlink capacity arbitrarily among the other nodes as long as the aggregate upload/download rates do not exceed the uplink/downlink capacity. Hereafter, we use the term fluid model to refer to the use of the Uplink Sharing Model along with the ability to infinitesimally divide link capacities. The provider $P$ is the originator of the live stream, it has an uplink capacity of $u(P)$. The set of clients subscribed to the live stream is $C$ of size $c=|C|$. Each client $c_{i} \in C$ has an uplink capacity of $u\left(c_{i}\right)$ and downlink capacity of $d\left(c_{i}\right)$. We denote the clients aggregate uplink capacity by $u(C)=\sum_{c_{i} \in C} u\left(c_{i}\right)$. The aggregate angels' uplink capacity is $u(A)$. We assume that the stream playout rate $r$ is constant. 2

Each client $j$ should be able to download fresh live content with a rate $x_{j}=\sum_{i \in C \cup A \cup P} x_{i j}$ greater than the playout rate $r$, where $x_{i j}$ is the rate between nodes $i$ and $j$. By definition the provider's upload bandwidth is not less than the playout rate $u(P) \geq r$, otherwise the provider cannot upload the live stream. Also, it is fair to assume that the downlink capacity of all clients is greater than the playout rate $d\left(c_{i}\right) \geq r \quad \forall c_{i} \in C$, otherwise those clients will not be able to play the live stream at the desirable playout rate.

\subsection{Optimal Angel Allocation}

In this subsection, we derive the minimum amount of angel uplink capacity needed in order for all clients to receive the live stream with rate $r$. First, we provide a lower bound on the angel uplink capacity, then find an optimal fluid allocation scheme achieving this bound.

Theorem 1. The minimum angel uplink capacity needed for all clients to receive the stream at a prescribed playout rate $r$ is:

$$
u(A) \geq \frac{c^{2}}{c-1} *\left(r-\frac{u(P)+u(C)}{c}\right)
$$

${ }^{2}$ It is recommended to use CBR for live streaming. But in the case of variable bit-rate encoding (VBR), we can use the optimal smoothing technique to achieve a constant bit-rate (CBR) 25. 
Proof. For a client to receive the stream live, its download rate should equal the playout rate. Thus, the slowest client should receive content with rate not less than $r$; $\min _{j \in C}\left\{x_{j}\right\} \geq r$. Because the average is always greater than the minimum, the average download rate should exceed $r$ as well; if $\min _{j \in C}\left\{x_{j}\right\} \geq r$ then $\frac{\sum_{j \in C} x_{j}}{c} \geq r$.

First, let us consider the case of no angels. The uplink sharing model dictates that the aggregate downlink capacity cannot exceed the aggregate uplink capacity in the swarm: $u(P)+u(C) \geq \sum_{\forall i \in C} x_{i}$. To optimally utilize $u(A)$ of the uplink capacity of the angels, an angel must download fresh data with a rate of at least $u(A) / c$ then upload it to all $c$ clients. Thus, in case of using angels, we have:

$$
\begin{aligned}
u(P)+u(C)+u(A) & \geq \sum_{\forall i \in C} x_{i}+\frac{u(A)}{c} \\
\frac{u(P)+u(C)+u(A)}{c}-\frac{u(A)}{c^{2}} & \geq \frac{\sum_{\forall i \in C} x_{i}}{c} \geq r
\end{aligned}
$$

Rearranging this inequality allows us to derive the angel uplink capacity needed to achieve the prescribed playout rate $r$.

$$
u(A) \geq\left(\frac{c^{2}}{c-1}\right) *\left(r-\frac{u(P)+u(C)}{c}\right)
$$

Not surprisingly, the bound in Theroem 1 suggests that the capacity of needed angels grows linearly with the number of clients and with the deficit between the playout rate and the client share of the provider and clients uplink capacities.

Theorem 2. All clients can achieve the playout rate $r$ when:

$$
u(A)=\frac{c^{2}}{c-1} *\left(r-\frac{u(P)+u(C)}{c}\right)
$$

Proof. We prove that the lower bound on the minimum angel uplink capacity is achievable by construction. Using a fluid model, we choreograph the transfer rates between nodes so as to achieve a download rate that equals the playout rate for all clients. The set of Equations 1 has those rates. The provider sends data to client $c_{i}$ with rate $x_{P i}$. The client $c_{i}$ in turn forwards this data to other clients $j \in C$ with rate $x_{i j}$. The provider sends data to the angel with rate $x_{P A}$, the angel relays this data to the $c$ clients immediately.

$$
\begin{aligned}
x_{P i} & =\frac{u\left(c_{i}\right)}{c-1}+\delta \quad \forall c_{i} \in C \\
x_{P A} & =\frac{u(A)}{c} \\
x_{i j} & =\frac{u\left(c_{i}\right)}{c-1} \quad \forall c_{i} \in C, i \neq j \\
\text { where } \quad \delta & =\frac{u(P)-r}{c-1} \geq 0
\end{aligned}
$$

These rates guarantee that each client receives data at rate $r$ without violating the uplink capacity constraint of any node. The aggregate download rate for client $j$ (from all sources) will be

$$
\begin{aligned}
x_{j} & =x_{P j}+x_{A j}+\sum_{i \in C, i \neq j} x_{i j} \\
& =\frac{u\left(c_{j}\right)}{c-1}+\delta+\frac{u(A)}{c}+\sum_{i \in C, i \neq j} \frac{u\left(c_{i}\right)}{c-1} \\
& =\frac{u(C)}{c-1}+\frac{u(P)-r}{c-1}+\frac{1}{c} * \frac{c^{2}}{c-1} *\left(r-\frac{u(P)+u(C)}{c}\right) \\
& =r
\end{aligned}
$$

The upload rate of each client, $i$, will not exceed its uplink capacity as $(c-1) * u\left(c_{i}\right) /(c-1)=u\left(c_{i}\right)$. The same can be said about the angels: $c * u(A) / c=u(A)$. Also, the aggregate upload rate from the provider will not exceed its capacity:

$$
\begin{aligned}
& x_{P A}+\sum_{j \in C} x_{P j} \\
& =\sum_{j \in C}\left(\frac{u\left(c_{j}\right)}{c-1}+\delta\right)+\frac{1}{c} * \frac{c^{2}}{c-1} *\left(r-\frac{u(P)+u(C)}{c}\right) \\
& =u(P)
\end{aligned}
$$

To ensure that each client receives non-duplicate data. The provider sends unique data to the angels. As for the clients, each client receives unique data with rate $u\left(c_{i}\right) /(c-1)$ and the same data with rate $\delta$ to all clients.

Figure 1 illustrates two examples of the optimal construction. The left side of Figure 1 is an example with three clients whose uplink capacities are sufficient to achieve a playout rate of $r$, thus there is no need for angels. Each client splits its uplink capacity between the other two clients. The provider sends data to clients with a rate that equals half their uplink capacity plus $\delta$. The $\delta$ part of the upload rate is identical, in terms of its content, to all clients, while the other part is unique, ensuring the uniqueness of data disseminated. On the right side of Figure 1 is an example where the uplink capacity of the provider and the two clients is not enough to secure a playout rate $r$. Thus, an angel is needed. Here, the angel downloads from the provider and uploads to the clients. Each client downloads from the provider and uploads some of what it receives from the provider to the other clients (and angels do not download from clients).
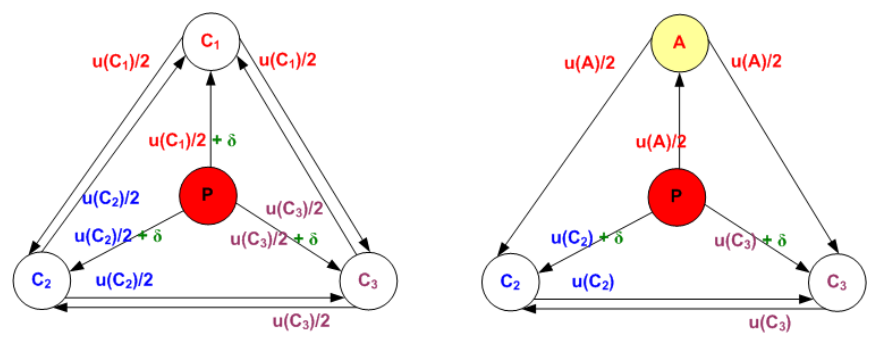

Figure 1: Illustrative examples of the optimal construction: 3 clients not in need of any angels (left) and 2 clients in need of one angel (right).

\subsection{Startup Delay}

In this section, we study the effect of packetization on startup delay. Assume that the unit of transfer is of size $\psi$. In the fluid model, $\psi$ approaches zero. In a practical setting, each node cannot forward a packet to another node before it finishes receiving it.

Theorem 3. Using the optimal construction in Theorem 2, the startup delay, $D$, is:

$$
D=\frac{\psi}{r}+\frac{\psi *(c-1)}{\min _{c_{i} \in C} u\left(c_{i}\right)}
$$

Proof. The proof is by construction. The delay until all clients receive a certain packet consists of two parts; the first is the delay until a client receives this packet from the 
provider $\psi / r$ and the second is the delay until it forwards the packet to all the other $c-1$ clients. The client that is forwarding the packet to all the other clients can be the one with the smallest uplink capacity, thus we divide the amount of data needed to be sent, $\psi *(c-1)$, by the minimum uplink capacity $\min _{c_{i} \in C} u\left(c_{i}\right)$.

Therefore, if the goal of the system is for all clients to enjoy an uninterrupted service, each client should fill a buffer of size $B$ before starting the playout, where:

$$
B=r * D=\psi+r * \frac{\psi *(c-1)}{\min _{c_{i} \in C} u\left(c_{i}\right)}
$$

This result suggests that the startup delay grows linearly with the packet size $\psi$ and with the number of clients $c$. Under the fluid model assumption, this is not consequential because $\psi=0$, resulting in a startup delay of zero as, $\lim _{\psi \rightarrow 0} D=0$. In practical settings however, $\psi \neq 0-e . g$., it could be the MTU of a TCP packet $\simeq 1,400$ Bytes. In such cases, the optimal construction may result in significant startup delays for large number of clients. In Section 3, we propose a dissemination strategy that achieves the desired download rate using minimum angels capacity while keeping the startup delay under a reasonable (logarithmic) bound.

\subsection{Implications on the Role of Angels}

The premise behind this work is that there is a significant gap between the clients' uplink and downlink capacities offered by ISPs. The promised higher download bandwidth encourages content providers to stream at an ever-increasing rate. Pure $\mathrm{P} 2 \mathrm{P}$ technology helps alleviate the cost on content providers by utilizing the clients uplink capacity. To bridge this gap, many peer-assisted file-based streaming constructions were proposed. Ours is the first to realize that it is more efficient for the added resources to download a fraction of the live stream instead of its entirety. Figure 2 illustrates the ecosystem of our angel-based content acceleration architecture. In this ecosystem, the aggregate downloaded data equals the aggregate uploaded data. The client uplink capacity cannot match the download rate of many streams. Thus, we need to add agents to this ecosystem that produce more than they consume. Angels provide that by downloading a fraction of the stream instead of consuming the already scarce resource of upload bandwidth by downloading unnecessary content. In live streaming, content providers do not

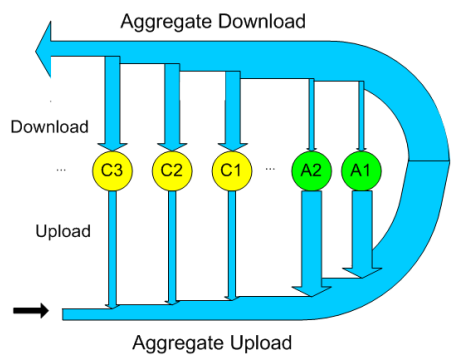

Figure 2: Our proposed ecosystem; clients download more than they can upload, and angels download as little as possible.

have the luxury of uploading the content to many servers beforehand. Thus, those servers will compete over the upload bandwidth with clients. In a peer-assisted streaming mechanism, like the one presented in 17], the system relies on more server capacity to stream at a higher stream rate not supported by the clients uplink capacity. Figure 3 shows a numerical analysis of the performance of angels against servers in a hypothetical setting. It compares the number of angels, each with uplink capacity ten, versus the number of servers, each with uplink capacity ten too, (on the $\mathrm{y}$-axis) needed to achieve a streaming rate (on the $\mathrm{x}$-axis). The results are shown for a family of curves with varying clients' ratios of uplink-to-playout rates - ranging from 3:10 to 9:10. The streaming rate (x-axis) varies between 1 and 9 . There are 100 subscribed clients and the number of clients an angel can connect with concurrently is 40 . The formula to compute how many servers we need is

$$
\frac{c * r}{u(a)-r} *(1-\text { ratio })
$$

and the formula for the number of angels is

$$
\frac{c * r * k}{u(a)(k-1)} *(1-\text { ratio })
$$

The growth in the number of angels is linear with the stream rate. However, if we use servers, the growth is superlinear. For example, when the streaming rate is 9 and the server upload capacity is 10 we need, nine-tenth of a server capacity is wasted in uploading the stream to another server (90\% overhead). The graphs compare different values for the ratio between the uplink capacity to the playout rate. The greater the gap, the more angels/servers the system will need. Clearly, angels are significantly more efficient than servers, especially when the stream playout rate is large.

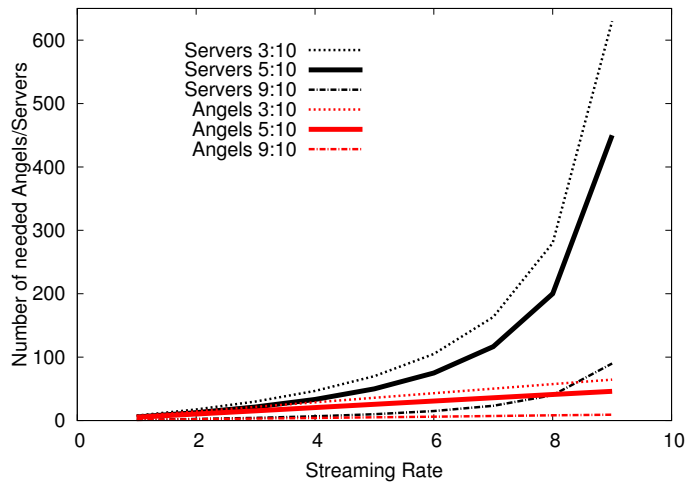

Figure 3: The number of angels versus the number of servers needed to achieve a certain playout rate for 100 clients.

Figure 4 shows the maximum number of clients that can download at a certain rate given the number of available angels/servers. We fix the ratio between the uplink rate and stream playout rate to 1:2. At low stream rates, a few high-capacity angels/servers can support many clients. As the stream rate increases, the number of clients satisfied by the service decreases. Again, the angels are more efficient as they are able to serve more clients consuming the same amount or resources.

\section{A PRACTICAL CONSTRUCTION}

The optimal construction in Theorem 2 requires each client to connect to all other clients. This leads to operational 


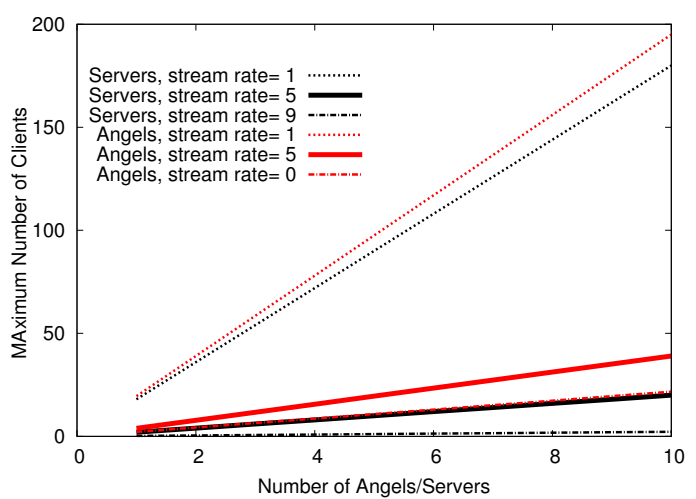

Figure 4: The number of clients that can be supported by a fixed number of angels or servers.

challenges and a start-up delay that grows linearly with the number of clients. To mitigate those problems we developed a new dissemination mechanism that constrains the node degree of each client to $k$, i.e., each clients can communicate with at most $k$ clients at any point in time. We call this construction AngelCast.

\subsection{Bounding the Angel Uplink Capacity}

The limit on the node degree influences angel effectiveness inversely. Ideally, an angel would download a small fraction of the stream and would upload it to all clients. Under a bounded-degree assumption, it is intuitive that we would need more angel capacity.

\section{Lower Bound on Angel Capacity}

Theorem 4. The minimum angel uplink capacity needed for all the clients to download at the playout rate, when each node is constrained to connect to only $k$ other nodes, is:

$$
u(A) \geq \frac{k * c}{k-1} *\left(r-\frac{u(P)+u(C)}{c}\right)
$$

Proof. Similar to the proof of Theorem 1 in order to optimally utilize $\mathrm{u}(\mathrm{A})$ of angel uplink capacity, an angel must download data at a rate of at least $\frac{u(A)}{k}$ then upload it to a maximum of $k$ clients, given that $k \leq c$. On the one hand, forwarding the same data to more than $k$ clients simultaneously would violate the out-degree constraint, and on the other hand, forwarding the same data to more than $k$ clients on stages would result in the reception of stale and out of stream data to some clients. Also, tearing down connections and building new ones frequently and systematically would result in performance degradation due to the nature of the transport protocols.

To ensure that the aggregate uplink capacity is more than the aggregate download rate:

$$
\begin{aligned}
u(P)+u(C)+u(A) & \geq \sum_{\forall i \in C} x_{i}+\frac{u(A)}{k} \\
\frac{u(P)+u(C)+u(A)}{c}-\frac{u(A)}{k * c} & \geq \frac{\sum_{\forall i \in C} x_{i}}{c} \geq r \\
\text { Thus: } u(A) & \geq\left(\frac{k * c}{k-1}\right) *\left(r-\frac{u(P)+u(C)}{c}\right)
\end{aligned}
$$

For small swarms, when $c \leq k$, the bounded-degree constraint is never reached, thus the bound on the angels up- link capacity does not change. Even when $c>k$ and $k$ is relatively large, we would not need significantly larger angel capacity as $c /(c-1) \simeq k /(k-1) \simeq 1$. For example if $k=100$ the overhead due to constraining the out-degree is around $1 \%$ even when the number of clients is extremely large.

\section{Construction Under a Bounded-Degree Assumption}

The optimal construction used in Theorem 2 assumes the ability of each client to connect to all other clients simultaneously. In the remainder of this section, we develop a practical construction under the constraint of limited node degree, $k$, where each node can communicate with only $k$ other nodes at any point in time.

Our construction divides the stream into $m$ substreams. We disseminate each substream using a multicast tree and each client subscribes to all the $m$ trees. The rate of dissemination of all the substreams, $r_{t}$ is equal, such that the sum of the substreams equals the playout rate, i.e., $r_{t}=r / m$. This construction is similar to what is done in Splitstream 10. and CoopNet 21.

The number of trees, $m$, depends on the allowed degree of any node, $k$. For $d$-ary trees, each node is connected to at most $d+1$ other nodes (one parent and $d$ children). Thus, the number of trees is bounded by: $m \leq\lfloor k /(d+1)\rfloor$. Choosing the arity of the trees is not trivial. On the one hand, choosing a small arity allows for a greater number of trees, each with a small substream rate. This will minimize the unassigned client capacities $\sum_{i \in C}\left(u\left(c_{i}\right) \bmod r_{t}\right)$ and utilizes angels more efficiently. As the theoretical results showed, angels are best utilized when they download the smallest substream which allows them to upload with their maximum bandwidth. On the other hand, choosing a larger arity would result in a smaller start-up delay, as we prove in Section 3.2 Also, choosing a large arity enables the utilization of the client with high uplink capacity in full because when a client subscribes to all trees as a parent of $d$ children, it can forward data with maximum rate $d * r_{t} * m=d * r$. Thus, any client uplink capacity above $d * r$ will be wasted. Consequently, choosing the right arity has to balance utilizing the resources and providing a small start-up delay.

Beside deciding on the number of trees and their arity, we need to decide on the placement of each client in each tree. First, let us consider the case, in which there are no angels. Adding angels to this construction is straightforward and will be explained shortly. In our construction, each client calculates how many children it can adopt across all trees, which equals the uplink capacity of the node divided by the rate of a substream, $r_{t}$. Let us call this parameter $l_{i}$, where $l_{i}=\left\lfloor u\left(c_{i}\right) / r_{t}\right\rfloor$. Our construction dictates that these children be allocated in the minimum number of trees. This is necessary to avoid degenerate trees, where parents have only one child. Thus, the number of trees where this client can have $d$ children is $g_{i}=\left\lfloor l_{i} / d\right\rfloor$. Client $i$ can have the remaining children assigned to one more tree and it will be a leaf in all the other trees. Whenever a new client arrives, it will join all trees. To ensure that no tree is starving for bandwidth while another one has an abundance of it. The new client will be a parent in the trees where there are fewer places to adopt more children. We use vacantSpots to denote the number of places in a tree where it is possible to adopt more children. 
The position of clients in each tree is equally important. To ensure a small start-up delay, the depth of the trees should be minimized. In a bounded-degree setting, the path from any node to the root should be logarithmic in the size of the swarm. Subsection 3.2 shows that full trees with large arity achieve that. Therefore, nodes that can adopt more children should be higher in the tree. Subsection 4.1 shows how we add/remove clients and change connections while maintaining low-depth trees.

Adding angels to this construction is straightforward. The minimum upload bandwidth of angels needed, $u(A)$, is given by the lower bound in Equation 4 This upload bandwidth would be divided equally between a number of angels $n_{a}=$ $u(A) /\left(k * r_{t}\right)$, each of which will be assigned to a different tree. Each angel will have $k$ children in that tree. Whenever the number of vacantSpots in a tree falls below a certain threshold, we know that this tree is in poor health, hence we add an angel to that tree. When a tree has too many vacantSpots, we eliminate an angel, if any exists in this tree. Even though when an angel is added, it is added to a single tree, the health of the other trees will also improve. This is because newer clients will not need to become parents in this tree and will allocate their resources to other needy trees.

As for the provider, it will be a parent to the roots of the trees. The excess capacity of the provider can be utilized fully as well, as the provider can adopt more children. To avoid adding angels in all trees at the start, the provider focuses its extra capacity in fewer trees.

To conclude, this construction allows each client to download with rate $r$ and achieves near optimal utilization of the clients uplink capacity. The remaining uplink capacity that is not enough to adopt a child equals $\sum_{i \in C}\left(u\left(c_{i}\right) \bmod r_{t}\right)$, which can be seen as insurance in the case of bandwidth oscillations. The gap between the clients' uplink capacities and the playout (and hence download) rate can be supplemented by angels, and each node will not have to connect to more than $k$ other nodes. In the following subsection, we will show that our construction has, on average, a logarithmic startup delay in the number of clients, $c$.

\subsection{Bounding the Startup Delay}

In this section, we will compute the startup delay given our construction in Subsection 3.1. A node with $d$ children dedicates $r_{t}$ of its uplink capacity to each one of them. Therefore, if we serialize the dissemination by sending a packet to a child at a time, the time to send a packet of size $\psi$ to the first child will be $\psi /\left(r_{t} * d\right)$. The time to disseminate a packet of size $\psi$ to all the $d$ children, is $d * \psi /\left(r_{t} * d\right)=\psi / r_{t}$ seconds. Each tree has $c$ children, thus, it has $\log _{d}(c)$ levels. Therefore, the time it takes for the last client in the last level of the tree to download a packet is:

$$
D=\log _{d}(c) * \frac{\psi}{r_{t}}=\log _{d}(c) * \frac{\psi}{r *(d+1) / k}
$$

This startup delay is the minimum when $\frac{\partial D}{\partial d}=0$

$$
\begin{aligned}
\frac{\partial D}{\partial d} & =\frac{\psi * k * \ln (c)}{r} *\left(\frac{-\left(\frac{d+1}{d}+\log (d) * 1\right)}{\ln ^{2}(d)}\right) \\
\ln \left(d^{*}\right) & =-\frac{d^{*}+1}{d^{*}} \quad \text { At } \frac{\partial D}{\partial d}=0
\end{aligned}
$$

The above means that in order to minimize the start-up delay, the degree of the tree should be maximized -i.e., $k-1$. This result illustrates the trade-off between minimizing the start-up delay and minimizing the needed angel capacity: The more trees we have, the better we are utilizing the angels/clients at the expense of increasing the start-up delay.

\section{ANGELCAST ARCHITECTURE}

Figure 5 shows the agents in our system. While the registrar does not disseminate data, it choreographs the connectivity of clients and angels in the system. The registrar is the main agent in our cloud service. Content providers contact the registrar to enroll their streams. The registrar uses the profiler to estimates the uplink capacity of clients. The accountant uses the estimated gap between the clients' uplink capacity and the stream playout bit-rate to give the content provider an estimate of how many angels it will need.

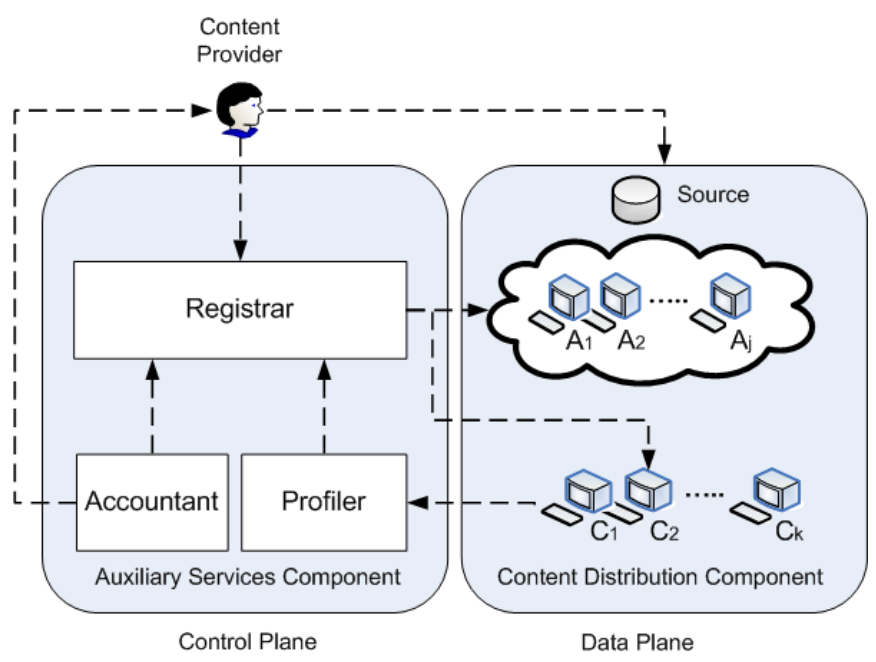

Figure 5: The architectural elements of AngelCast.

\subsection{Membership Management: The Registrar}

Live streaming swarms are dynamic in nature. Clients arrive and depart the stream constantly. Also, some bilateral connections between clients can degrade arbitrarily. Thus, it is absolutely essential to incorporate resilience in the design of swarms to ensure that some minimal quality of service is maintained. In this section, we explain how AngelCast handles membership management, i.e., handling client arrival and departure, and replacing degraded connections with new ones. Our system relies on a special server to achieve that, the registrar. The registrar is a special node in the system that orchestrates the swarm and choreographs the connectivity of the clients. When a new node joins the stream, it contacts the registrar and informs it of its available upload bandwidth. The registrar uses a data-structure representing the streaming trees and assigns the new client to a parent node in each tree. The registrar also decides how many future children the new node can adopt in each tree. Clients can also "complain" to the registrar about their parents. The registrar would pick a new parent for a complaining client, informs the client of its new parent, and also probes the underperforming parent to ensure it is still alive. If not, it pro-actively informs other children to disconnect from it and provides them with new parents.

These decisions are crucial in guaranteeing a continued service with low start-up delay and little disruption. In or- 
der to ensure fast response to clients' requests, the registrar maintains a data-structure containing the state of each node in the system. The state of a node contains: (1) the depth of the subtree rooted at that node, (2) a pointer to the closest descendent with a vacant spot that can adopt one new child, which we call the closestAdopter, and (3) the distance to the closestAdopter. When a new client joins the swarm, the registrar adds it to the root's closest adopter in each tree. The arrival and departure of clients changes this state. Algorithm 1 shows the function that updates the closestAdopter as well as the distance to it. The value of the closestAdopter can change for the new/old parent as well as for predecessors, recursively all the way to the root (by construction, a logarithmic process at worst). If the node has vacantSpots, then it is its own closestAdopter with distance zero. Otherwise, it picks the closestAdopter of one of its children, the one with the minimum distance to its closestAdopter. The update will propagate recursively along the path towards the root until the closestAdopter of a node along the path does not change.

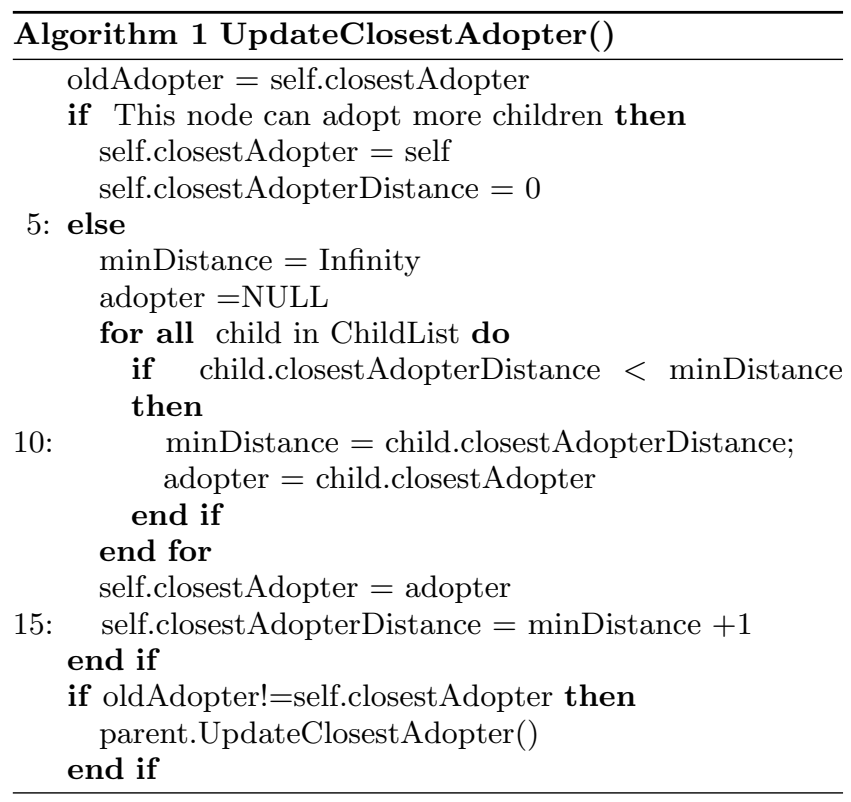

Figure 6 illustrates how the value of the closestAdopter changes when a new client arrives. Before the addition, nodes $\mathrm{C}$ and $\mathrm{D}$ had vacantSpots. The root, node $\mathrm{A}$, has node $\mathrm{C}$ as the closestAdopter. Thus node $\mathrm{C}$ will adopt the new node, $\mathrm{F}$. This will change the value of the closestAdopter up the path to the root. Nodes C, F will have no closestAdopter. Thus, node A's closestAdopter will become node D, its other child, node B's, closestAdopter.

As we alluded before in Subsection 3.2 , in order to minimize the average start-up delay, we need to minimize the depth of the tree. We note that the degeneration of a tree could be caused by those few nodes that cannot have the maximum number of children. Our goal is to push those nodes down the tree to avoid this condition. In order to do so, we allow new nodes to intercept certain connections. By intercepting a connection we mean severing a connection between a parent and a leaf child node, making the parent adopt the new node, and making the new node adopt the child node. We prefer interception over adoption if the distance to the closestIntercept is smaller than the distance

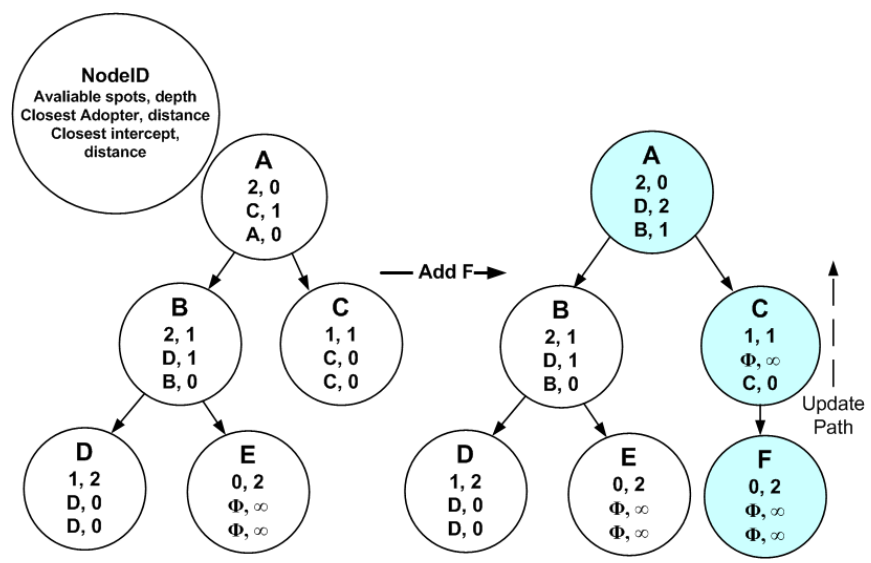

Figure 6: Node $F$ joins the tree, the registrar updates the data structure accordingly.

to the closestAdopter. We maintain and update the information about the closestIntercept and its distance for each node in the tree in a way similar to the closestAdopter.

The registrar receives complaints from nodes about their parents when they are not downloading at an adequate bitrate. The registrar sends a probe to the parent, if the parent is alive and replies, the problem is with the link not with the parent. The registrar severs the connection to that parent and attaches the complaining child, and the subtree below it, to the closestAdopter in the tree. We need to ensure that the complaining node is not re-attached to the same parent, or worse to a descendent of its own. We ensure that by setting the closestAdopter distance of the parent to a very big number and propagating the update upward the tree, forcing the root to choose a closest adopter away from the complaining node. We then attach the severed subtree to the new closestAdopter then set the closestAdopter distance of the old parent to zero and update up the tree. By the end of this process, the complaining node gets allocated, with its subtree, to a different part of the tree and the values of closestAdopter of all the nodes are adjusted.

If the registrar's probe to a node results in no response, the registrar concludes that the client unsubscribed from the stream. Thus, it will actively remove it from all trees. When a node is removed from a tree, each of its orphaned children will be added, one by one, to the closestAdopter in the tree. To maintain balance in the tree, the children with smaller distance to their closestAdopter are added before the children with larger distance to their closestAdopter. Our service enables a graceful departure of clients by allowing them to declare their intention to leave the stream.

Figure 7 illustrates how our membership management techniques work through an example. Step 1 shows the initial state in which the provider is the root of the three trees, with an uplink capacity of 4 and a stream playout rate of 3 . Thus, the root, s, has 4 vacantSpots, two of which are assigned to the first tree and one vacantSpot for each of the second tree and the third tree. Client $x$ joins in Step 2. It has two vacant spots, both of them will be assigned to the second tree. As we discussed before we assign a client as a parent in the minimum number of trees to maintain low depth trees. As a result of client's $x$ arrival, the number of vacantSpots in the third tree is reduced to zero. Thus a new angel, $A$, is added 
automatically to the third tree. The angel's uplink capacity equals 3 , allowing it to have three children in one tree. If we had only one tree, the angel would have been useless as it will have had only one child and downloading as much as it is uploading. Because there are no vacantSpots in the third tree, the added angel will intercept the connection between the provider and client $x$. Step 3 illustrates the arrival of client $y$. Both of its vacantSpots will be assigned to the tree with the minimum number of vacantSpots, which is tree 1 . In Step 4, client $x$ complains about its connection to the provider in the first tree. The registrar disconnects it from the provider and instructs it to connect to client $y$. Step 5 illustrates the departure of client $y$. It will be removed from all trees and its children, if any, will be added one by one.

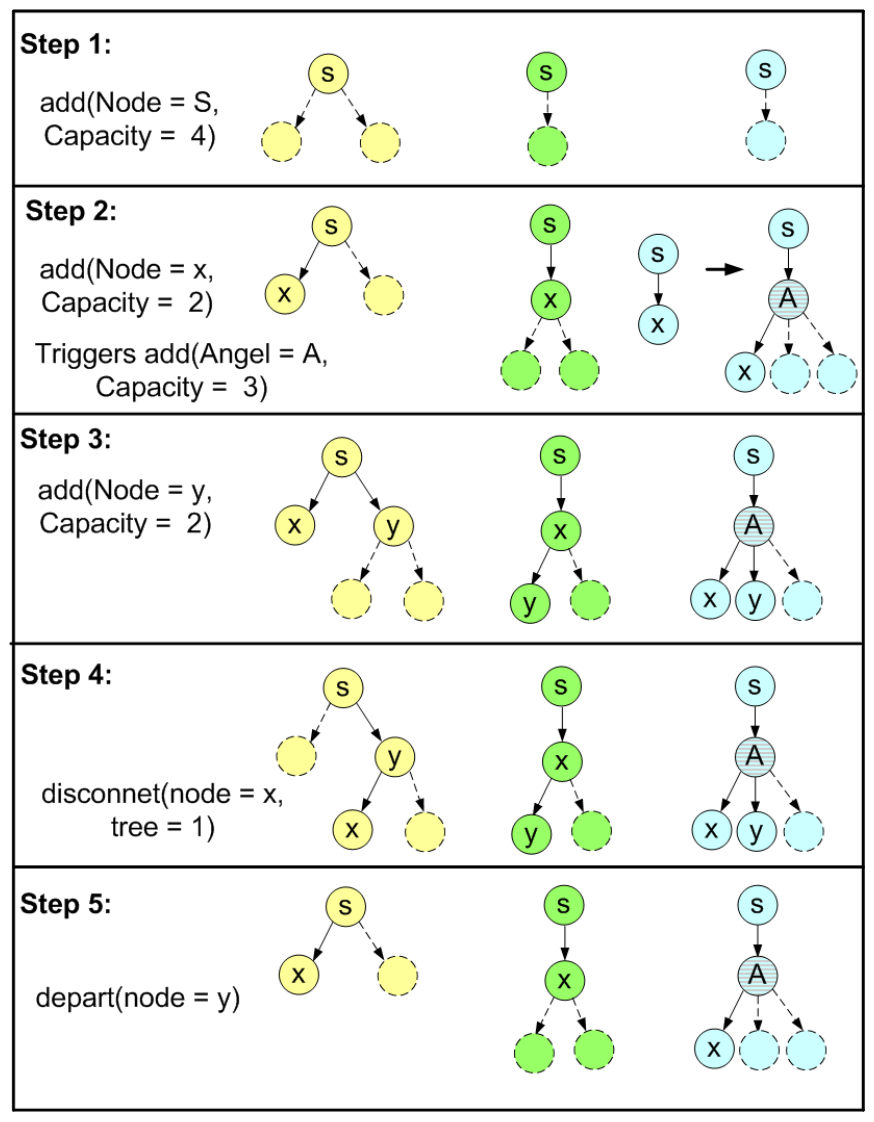

Figure 7: A hypothetical scenario illustrating the formation of AngelCast trees when clients join, leave or change parents.

\subsection{The AngelCast Protocol}

The registrar decides on the number of substreams, the fan-out of the trees and adds the provider as root to all trees. It also initializes the data structure in which the state of the system is kept. The registrar then starts a listener process to receive join requests and complaints from clients. It uses the membership management techniques, described in Subsection 4.1 to respond to such request. Whenever the number of vacantSpots in a tree falls below a certain threshold, the registrar instantiates a new machine from the cloud as an angel. When a tree has too many vacantSpots, an angel is released from this tree, if any exists. A full implemented system, serving many live streams concurrently, can instantiate a couple of machines and leave them on standby at all time. Therefore, in the case when a stream is in need of help, an angel would be ready to help and there would be no need to wait for the typical delay associated with acquiring a machine from the cloud.

In our implementation, the provider and clients need to download plug-ins (software) to enable them to interact with the AngelCast system. On the provider side, the software divides live content as it arrives into substreams, which are maintained in separate substream buffers. The content of each buffer is divided into chunks of fixed size. The provider also starts a listener process, which instantiates threads in response to join requests. These threads read from a substream buffer and sends chunks of data to the client. On the client side, the software starts by contacting the registrar asking to join a stream. The registrar replies with information about the stream as well as the identity of a parent capable of serving the (sub)stream in each tree. Upon contacting these parents, a newly-arriving client is able to start downloading the substreams into different buffers. The software on the client side includes a thread that reads from these buffers in a round robin fashion and writes to a local port using an HTTP server. Any media players with the ability to play streams over HTTP (e.g., mplayer) can play out the stream from this local port. Similar to the software on the provider side, the software at the client also starts a listener process, which instantiates threads in response to join request from children. When such a request is received for a specific substream, the software sends data from the buffer associated with that substream. The angels' software is similar to the software at the clients, except that angels need only subscribe to one substream and then listen and serve incoming client requests for that one substream.

Figure 8 illustrates an example of the interaction between a newly arriving client, client $A$, the registrar and other clients chosen by the registrar as parent to client $A$ in two trees. Client $A$ joins the system by sending a "Join" message (message \#1) to the registrar, the "Join" message contains the ID of the requested stream and the uplink capacity it is willing to contribute. The registrar replies with a "Welcome" message (message \#2) informing the client with the stream playout rate, the number of trees (substreams), the chunk size, and the identity of the chunk it should download first. The registrar also sends to the client the IP/port\# of each parent that the client should contact for each substream (Messages \#3 and \#5). When contacting these parents (messages \#4, \#6), the client specifies the tree ID and the chunk it is expecting to start downloading from. Messages \#7 to \#13 represent data messages from the parents to client $A$. The data messages has the chunkID and the associated streaming data. In this example, the streaming continues smoothly (message \#13), after that the connection is lost or degraded (message \#14 is lost). The client realizes that and contacts the registrar before it depletes its buffer. It sends message \#15 to the registrar informing it that it was disconnected from its parent in the first tree, client $B$. The registrar replies to client $A$ with a new parent to connect to, client $D$ (Message \#16). The client sends message \#17 to the new parent, client $D$, requesting chunks starting at where it stopped, the new parent starts streaming this substream. In the meanwhile, the registrar probes 
old parent, client $B$, to check if it is still alive or not (Message \#18). In this case it receives an "EchoBack" message (\#19), and the registrar sends the old parent message \#20 informing it to disconnect that child. If the registrar had not received an "EchoBack" message, it would have assumed the client is disconnected and would have sent a proactive message to all its children in all trees to connect to a different parent. For security reasons, the registrar sends messages to the parents informing them which clients to accept as children. The client can ignore any download request from any unauthorized client. We omitted those messages from this example for simplicity.

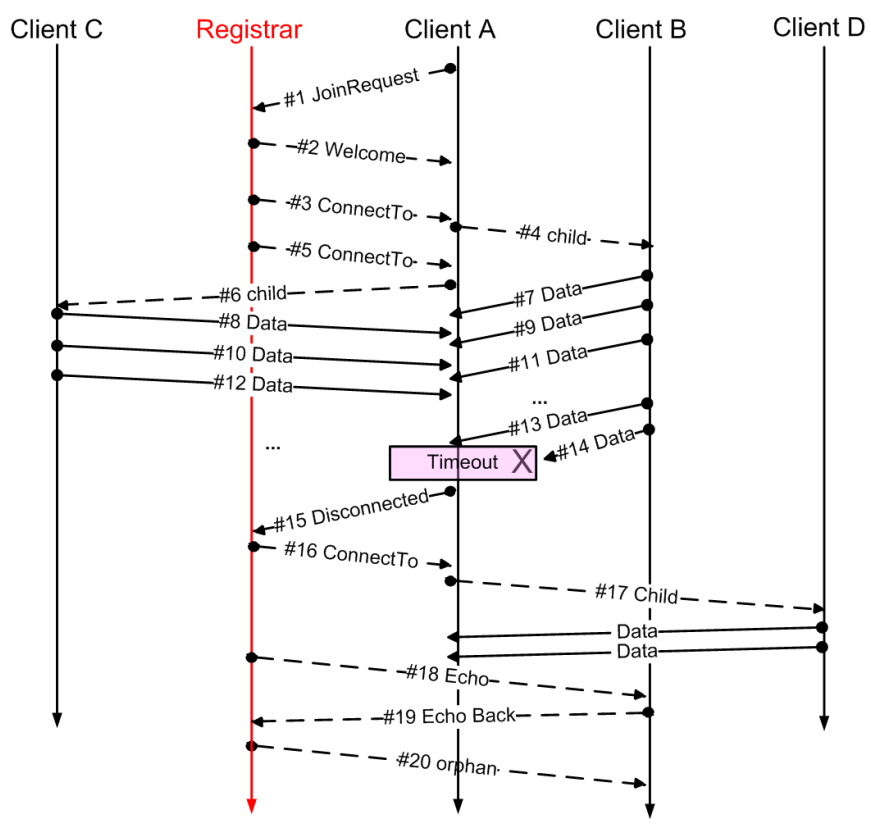

Figure 8: An interaction diagram showing the exchange of messages between a new client, the registrar and the parents.

We implemented a prototype of our AngelCast protocol in python ${ }^{3}$ Our prototype includes the code for the registrar, provider, clients and angels. Our prototype does not include the profiler, thus clients report how much uplink capacity they are willing to contribute to each swarm.

\section{EXPERIMENTAL EVALUATION}

To evaluate the performance of our AngelCast prototype, we deployed it in the widely used research platforms: Emulab 6 and PlanetLab 7 . On the one hand the Emulab experiments give us accurate insights by isolating our protocol form other experiments, and also making it possible for our results to be repeatable. On the other hand the PlanetLab experiments are meant to validate that AngelCast performs well "in the wild" on the Internet.

Our main motivation in performing these experiments is three-fold: (1) establish confidence in our implementation by comparing its performance to that of widely used streaming solutions, (2) establish the effectiveness of deploying angels from the cloud for the purpose of guaranteeing certain

${ }^{3}$ The code is available at: http://cs-people.bu.edu/ remos/research/angels.html streaming rates, and (3) measure the performance of our system under churn. We deploy the registrar on a machine of its own. The angels were also deployed on Emulab/PlanetLab machines instead of the cloud.

The first set of experiments aims at validating our AngelCast prototype by comparing its performance to that of SopCast 5 . SopCast is a popular P2P streaming client used widely on the Internet. We ran SopCast and AngelCast protocols on the same set of machines at the same time to neutralize unpredictabilities related to host/network processes (e.g., cross-traffic).

We performed experiments to compare the frame dropratio of AngelCast vs Sopcast for streams of varying rates (280Kpbs to $1.4 \mathrm{Mbps}$ ) on Emulab. The result: 4 are shown in Figure 9a. Emulab machines tend to have significantly higher uplink bandwidth than the average household. Thus we use traffic shapers to limit the uplink capacity of the nodes. We limit the uplink capacity of the provider to twice the stream rate, $2 * r$, and the thirty two clients uplink capacity to $(4 / 3) * r$. This assignment guarantees that there is enough upload bandwidth for all clients, thus, there is no need for angels. The frame drop-ratio of AngelCast and SopCast are comparable when the upload bandwidth is plentiful.

Figure 9p shows the result of the same previous experiment on PlanetLab. The frame drop-ratio is significantly higher than in Emulab but the performance of AngelCast and Sopcast is still comparable. Because Emulab allow us to perform repeatable experiments and to isolate the performance from other experiments running on the same machine, we decided to run the rest of the experiments on Emulab.

The second set of experiments aims to characterize the effectiveness of angels. Our system deploys angels when a tree has no vacantSpots. We secured 26 clients for downloading a live stream at $r=950 \mathrm{Kbps}$ playout rate. We vary the client uplink capacity between $60 \%$ to $100 \%$ of the stream rate, $r$. AngelCast splits the stream into ten trees each with a fanout of three. The provider's upload bandwidth is double the stream rate, ensuring that the provider is not the bottleneck. An angel uplink capacity is 1.5 times the stream rate, ensuring that it is larger than the stream rate, but also that it does not have too many children in one tree (maximum $=15)$.

On the $\mathrm{x}$-axis of Figures $10 \mathrm{a}$ and $10 \mathrm{p}$, we vary the client uplink capacity, shown as the ratio between the client uplink capacity and the stream rate. On the y-axis of Figure 10b we plot the capacity of angels deployed by AngelCast against the theoretical bound for the minimum angel capacity (AngelCast theoretical). Also, we compare it against the minimum server capacity when the server downloads the whole live stream (ServerCast). The results confirm that AngelCast utilizes near minimal capacity, and that it achieves significant savings when compared to ServerCast. On the left-hand-side of Figure $10 \mathrm{a}$ (in red), we plot the angel capacity being used and on the right-hand-side scale (in black), we plot the associated frame drop ratio. This experiment verifies that our system achieves reliable streaming utilizing near minimal resources.

The third set of experiments aims to demonstrate the performance of AngelCast under churn. In live streaming, churn is due to client arrivals and departures. This is different from

\footnotetext{
$\overline{{ }^{4} \text { All results are shown within }}$ 95\% confidence interval.
} 

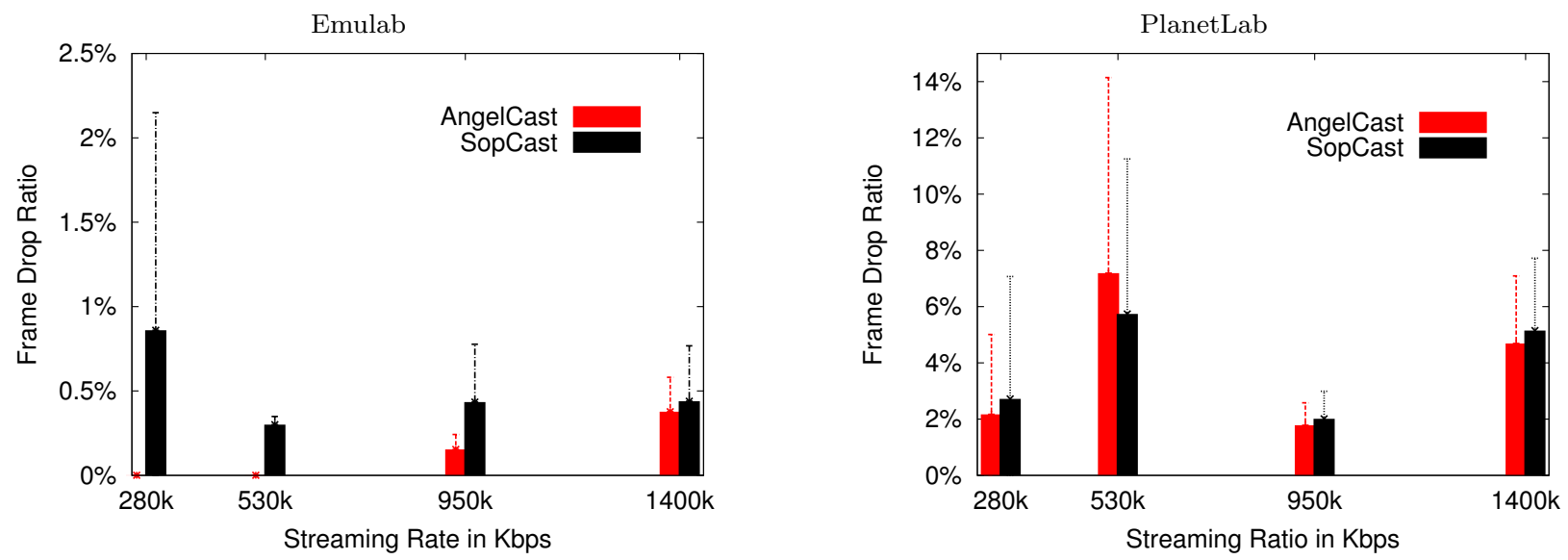

Figure 9: The frame drop ratios of AngelCast vs SopCast.
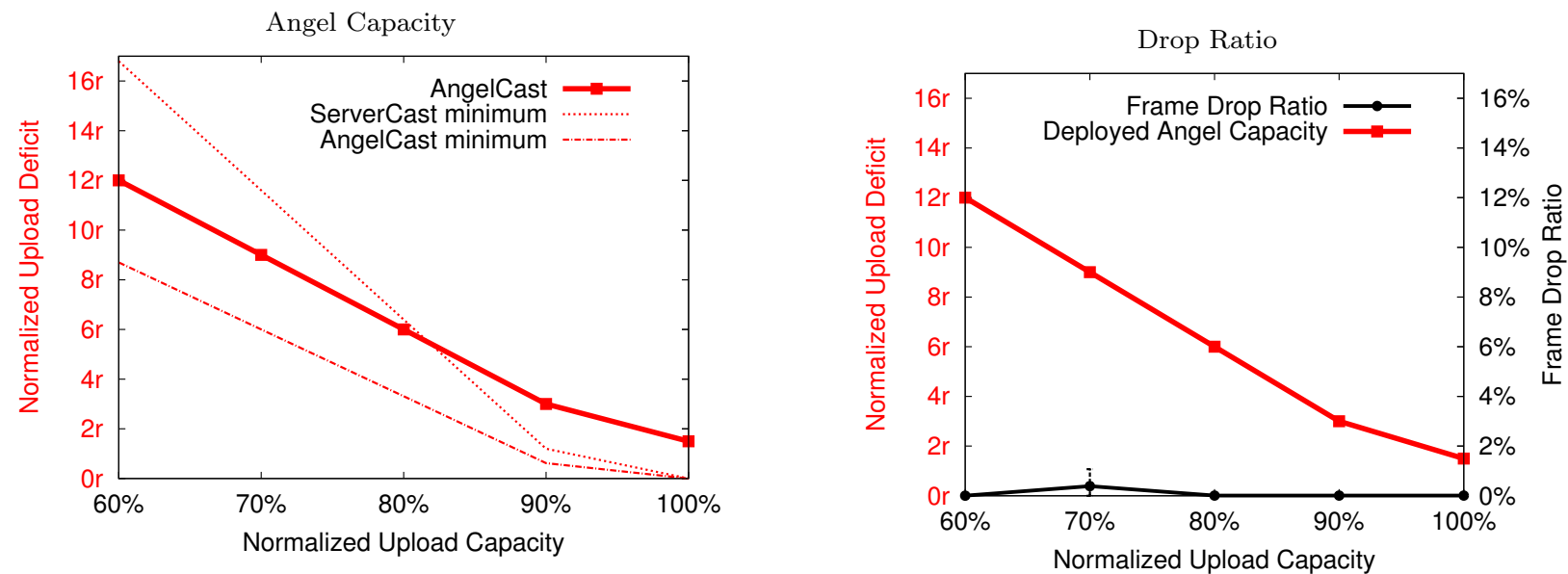

Figure 10: Minimal amount of angel capacity is sufficient to achieve good stream rates.

how churn is typically modeled in VoD where playback functionality, such as pause, seek and fast-forward must be considered as well 14 . Therefore in this experiment, we observe a live stream over a period of 210 seconds, whereby clients join the stream at a constant rate of 0.1 arrival per second (i.e., one arrival every 10 seconds on average). Clients stick to the stream for an exponential amount of time of mean 50 , 100,150 or 200 seconds then leave.

We set the uplink capacity of the provider to be twice the playout rate; we set the uplink capacity of angels to be $150 \%$ the playout rate; and we set the clients' uplink capacity to be $70 \%$ the playout rate. On the $\mathrm{x}$-axis of Figure 11 we vary the expected duration of the client's stay. On the right y-axis we show the frame drop-ratio. This experiment shows that, as expected, higher churn results in poor performance, (e.g. when clients stay less than a minute on average, the frame drop ratio is as bad as $25 \%$ ). On the left y-axis, we plot the aggregate capacity of the deployed angels. When there is no churn, six angels are needed to fill the capacity gap. In the presence of churn, the number of deployed angels is almost fixed to ten, the number of trees. The reason for that is the lack of vacantSpots in each tree at different points during the experiment, due to churn.

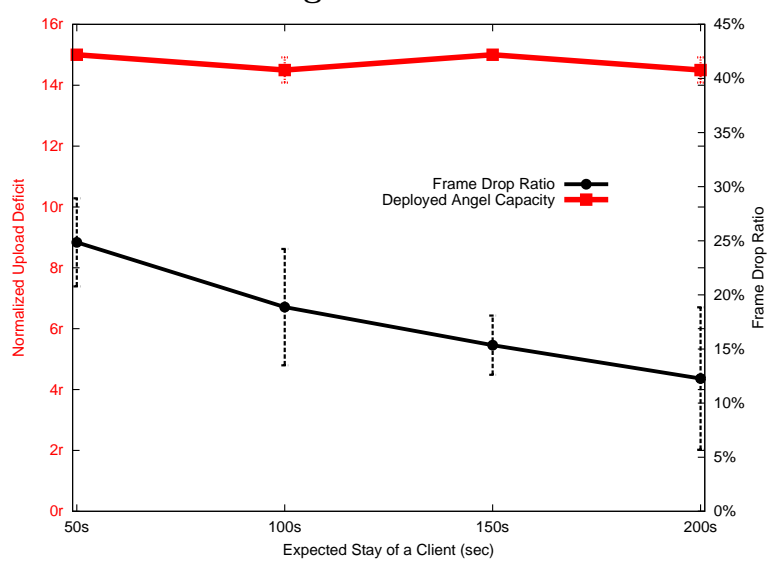

Figure 11: The performance of AngelCast under churn.

\section{RELATED WORK}

This paper builds on a rich body of work that approach the problem of content delivery in general and live streaming in particular from a number of perspectives:

Pull-based Mesh Protocols: There is a large number of papers/systems that utilize pull-based mesh streaming, such as SopCast, PPLive, UUsee, Joost, and CoolStream- 
ing. Our push-based approach differs from these in that it choreographs the connectivity among nodes to guarantee the quality of streaming for every client. We choose to compare AngelCast against SopCast [5] as it is extensively used, allows users to stream their own channels and works with mplayer over Linux. An example of such pull-mesh protocols is CoolStreaming 29], the streaming version of Bittorrent. The difference is that the deadline of a chunk playtime is a factor in the piece selection algorithm. Feng et. al. 13 illustrated the inherent shortcomings of pullbased mesh networks as well as providing a glossary of such protocols.

Peer-Assisted Content Distribution: The research community is aware of the promise of $\mathrm{P} 2 \mathrm{P}$ in alleviating the load of content distribution on servers. Nonetheless, it is also aware of its limitations, especially in providing sufficient upload bandwidth. For file sharing, Wang et al 28 proposed adding powerful peers to BitTorrent swarms to accelerate the download rate. This is not optimal, because these "helpers" will unnecessarily consume the scarce upload bandwidth in the swarm. In Antfarm 23], the authors propose a protocol for seeders to measure the vital signs for multiple swarms and allocate more seeder bandwidth to struggling swarms. Our previous work 27 showed that choreographing the connectivity of a swarm and using angels instead of seeders results in minimizing the maximum client download time for a fixed group of clients in a swarm. Jin el. al. 15 introduced edge caching to help original servers stream to clients at the prescribed bit-rate. The cache contains the objects where the ratio between the client request rate and the deficit between download rate and playback rate is maximal. This work is different as it requires in-network caching, but it highlights the need for infrastructure help to ensure smooth streaming.

Modeling P2P Performance: Qiu and Srikant's seminal work 24 is the first to characterize the average download time in a swarm. Das et al 12 extended Qiu's model 24 of swarm download rate to incorporate seeders. The result points out that the average download time is inversely proportional to the seeders' aggregate upload rate. However, the increase in the number of peers requires a linear increase in the number of seeders to maintain the same average download time. Although Qiu's model is fundamentally different, these results are in line with our findings. Parvez et al. 22 extended Qiu and Srikant's model 24 to the case of stored VoD, studying the need for sequential progress instead of random chunk download. This is inline with our design concept of choreographing the connectivity of the swarm instead of relying on random chunk download.

Kumar et al. 16 used the uplink sharing model 18 to find the bound on the highest streaming rate a swarm can handle given the uplink/downlink capacities of the clients. They proposed a fluid construction that achieve their bound. We extend their model to compute the required angel capacity to achieve a certain streaming rate. Likewise, we built an optimal fluid construction that achieves the bound, incorporating angels. They highlighted the gap between the uplink capacity and download rate, stating that most channels in PPLive are running at rate 2-4 times the uplink capacity of many residential broadband peers. Angels are the answer to this problem. Liu et al. 17] extended Kumar's model in the case of bounded node degree as well. They proposed creating spanning trees with varying streaming capacities. Given their construction, they provided bounds on the depth of the tree, the maximal upload rate and the minimum server capacity. Their constructions assumes the provider can connect to all clients, that the number of spanning trees can reach the number of clients and the depth of some trees can be linear in the size of the swarm. Our AngelCast technique avoids those three problems.

Multicast Multi-Tree Construction: Many papers leverage the idea of constructing multiple multicast trees (a forest) for file distribution as well as for streaming. SplitStream 10. constructs a forest of multicast trees, one for each stripe, all with the same rate. This approach is different from ours in that the client can choose the number of stripes it wants to subscribe to, thus receiving a subset of the broadcasted data. In contrast to tree-based multicast, the load on the nodes is balanced as each node is an internal node in one tree but a leaf node on the others. When the internal nodes of one tree can not adopt any more children, the child must search for some node in the excess capacity tree to download this strip. This leads to inefficiencies as a node could perform a distributed linear search until it finds a suitable parent. More importantly a client with significantly large capacity can adopt many children each in a different tree resulting in degenerate trees and more than logarithmic depth of trees. Our technique makes sure that any parent has at least two children except for the nodes in the second to last level, insuring a logarithmic depth of all trees.

P2PCast 19] is a modification of SplitStream where all nodes subscribe to all trees to download the same content. They require from each client to participate an upload bandwidth equal to the download rate. This could be unrealistic given the diversity of user's uplink capacities and the shielding of some clients behind NATs. CoopNet 21, 20 creates multiple trees, each streaming a substream of an MDC encoded video. There contribution is in providing a mechanism to cope with the fluctuation in the available bandwidth.

Coding for Adaptive Streaming: Multiple Description Coding (MDC) 11 and Scalable Video Coding (SVC) 9 were introduced to enable clients to download the same video on different rates/qualities. MDC tends to be more theoretical, where any number of descriptions are enough to decode the movie at a rate proportional to the number of received descriptions. SVC codes the video in layers, the base layer is essential for decoding while the subsequent layers are increasingly less important. Such techniques are increasingly deployed to overcome the uncertainty of the available bandwidth and its fluctuation, in particular Dynamic Adaptive Streaming over HTTP (DASH) 26. We consider those techniques complementary to our angels approach. Providers who prefer better than best effort delivery to their clients can deploy angels to offer better download rate. MDC and SVC can be deployed in conjuncture with angels in this case, where clients can attempt subscribing to $m$ trees downloading $m$ descriptions/layers. If a client is not able to download all the layers, it is still able to decode the video with lower quality. Our current prototype implementation works over HTTP, we are planning on extending our protocol to conform more closely to DASH. 


\section{CONCLUSION}

In this paper we considered the potential of peer-assisted content distribution for affordable high-quality live streaming. As, the deficit between clients' uplink and downlink capacities limits the use of pure $\mathrm{P} 2 \mathrm{P}$ architecture in such a setting, we introduced the notion of angels - servers who do not have a feed of the live stream and are not interested in downloading it in full. We computed the minimum amount of angel capacity needed in a swarm to achieve a certain bit-rate to all clients and provided a fluid model construction that achieves that bound. We introduced practical techniques that handle limited node degree constraints and churn. We built AngelCast, a cloud-based service that assists content providers in delivering quality streams to their customers, while allowing the content providers to leverage the customers' resources to the fullest. We deployed AngelCast unto two research platforms: Emulab and Planetlab. We showed that the performance of AngelCast is comparable to that of SopCast, a widely used streaming protocol, and that it is capable of supplementing the bandwidth deficit with near minimal capacity, while being able to handle churn. We are currently developing a version of AngelCast for wide-spread deployment. We are planning on conducting extensive evaluation and on collecting measurements of its performance in delivering real-world live streams. Our future work will explore ways in which underutilized angels are managed. This includes the possibility of using angels across multiple swarms. Security is another focus of our future work, especially securing the registrar control messages.

\section{REFERENCES}

[1] Akamai Netsession. http://www.akamai.com/client/

[2] Octoshape. https://www.octoshape.com/

[3] Pando Networks. http://www . pandonetworks.com/cdn-peering

[4] Bittorrent DNA. http://www.bittorrent.com/dna/technology

[5] SopCast: Free P2P Broadcasting. http://www. sopcast.org/.

[6] Emulab. https://www.emulab.net/

[7] PlanetLab. https://www.planet-lab.org/

[8] Netflix Tech Blog 2010. http://techblog.netflix.com/2011/01/ netflix-performance-on-top-isp-networks.html

[9] Abboud, O., Zinner, T., Pussep, K., Sabea, S. A., And Steinmetz, R. On the impact of quality adaptation in SVC-based P2P video-on-demand systems. ACM 2nd MMSys '11, pp. 223-232.

[10] Castro, M., Druschel, P., Kermarrec, A., Nandi, A., Rowstron, A., And Singh, A. Splitstream: high-bandwidth multicast in cooperative environments. In SOSP'03.

[11] Chou, P., Wang, H., and Padmanabhan, V. Layered multiple description coding. In Proc. Packet Video Workshop (2003), Citeseer.

[12] Das, S., Tewari, S., and Kleinrock, L. The Case for Servers in a Peer-to-Peer World. Communications, 2006 IEEE International Conference on.

[13] Feng, C., Li, B., And Li, B. Understanding the performance gap between pull-based mesh streaming protocols and fundamental limits. In INFOCOM 2009 , IEEE (2009), IEEE, pp. 891-899.

[14] Guo, Y., Yu, S., Liu, H., Mathur, S., AND RAMASWAMY, K. Supporting vcr operation in a mesh-based p2p vod system. In $C C N C$ 2008. 5th IEEE (2008), IEEE, pp. 452-457.

[15] Jin, S., Bestavros, A., And Iyengar, A. Accelerating internet streaming media delivery using Network-Aware partial caching. ICDCS'02, 153+.

[16] Kumar, R., Liu, Y., And Ross, K. Stochastic fluid theory for P2P streaming systems. In INFOCOM 2007. 26th IEEE International Conference on Computer Communications. IEEE (2007), IEEE.

[17] Liu, S., Zhang-Shen, R., Jiang, W., Rexford, J., And Chinng, M. Performance bounds for peer-assisted live streaming. In ACM SIGMETRICS Performance Evaluation Review (2008), vol. 36, ACM.

[18] Mundinger, J., Weber, R., And Weiss, G. Optimal scheduling of peer-to-peer file dissemination. Journal of Scheduling 11, 2 (2008), 105-120.

[19] Nicolosi, A., And Annapureddy, S. P2pcast: A peer-to-peer multicast scheme for streaming data. In 1st IRIS Student Workshop (ISWŠO3). Available at: http: //www. cs. nyu. edu/nicolosi/P2PCast.ps

[20] Padmanabhan, V., Wang, H., and Chou, P. Supporting heterogeneity and congestion control in peer-to-peer multicast streaming. Peer-to-Peer Systems III (2005), 54-63.

[21] Padmanabhan, V. N., Wang, H. J., and Chou, P. A. Resilient peer-to-peer streaming. In 11th IEEE ICNP'03 (Nov. 2003), IEEE Comput. Soc.

[22] Parvez, N., Williamson, C., Mahanti, A., And CARlsson, N. Analysis of bittorrent-like protocols for on-demand stored media streaming. SIGMETRICS'08.

[23] Peterson, R. S., And Sirer, E. G. Antfarm: efficient content distribution with managed swarms. In NSDI'09, USENIX Association.

[24] Qiu, D., And SRIKant, R. Modeling and performance analysis of BitTorrent-like peer-to-peer networks. SIGCOMM Comput. Commun. Rev. 34 (2004).

[25] Salehi, J., Zhang, Z., Kurose, J., And Towsley, D. Supporting stored video: Reducing rate variability and end-to-end resource requirements through optimal smoothing. ACM SIGMETRICS Performance Evaluation Review 24, 1 (1996), 222-231.

[26] Stockhammer, T. Dynamic adaptive streaming over http-: standards and design principles. In $A C M$ 2nd MMSys'2011.

[27] Sweha, R., Ishakian, V., and Bestavros, A. Angels In The Cloud: A Peer-Assisted Bulk-Synchronous Content Distribution Service. In IEEE CLOUD'2011: The IEEE International Conference on Cloud Computing.

[28] Wang, J., Yeo, C., Prabhakaran, V., and RAMCH, K. On the role of helpers in peer-to-peer file download systems: Design, analysis and simulation. In In IPTPS'07 (2007).

[29] Zhang, X., Liu, J., Li, B., And Yum, T. Coolstreaming/donet: A data-driven overlay network for efficient live media streaming. In proceedings of IEEE Infocom (2005), vol. 3, Citeseer, pp. 13-17. 